CrossMark \& click for updates

Cite this: J. Mater. Chem. C, 2015, 3, 6240

\section{Enhancing the photovoltaic properties of terpolymers containing benzo[1,2-b:4,5- $b$ ']- dithiophene, phenanthro[4,5-abc]phenazine and benzo[c][1,2,5]thiadiazole by changing the substituents $\dagger$}

\author{
Qunping Fan, ${ }^{a}$ Yu Liu, ${ }^{* a}$ Manjun Xiao, ${ }^{a}$ Wenyan Su, ${ }^{a}$ Huishan Gao, ${ }^{a}$ Jianhua Chen, ${ }^{a}$ \\ Hua Tan, ${ }^{a}$ Yafei Wang, ${ }^{a}$ Renqiang Yang*b and Weiguo Zhu*a
}

\begin{abstract}
In this study, three novel donor-acceptor (D-A)-type random conjugated terpolymers of PBDTT-PPzBT-H, PBDTT-PPzBT-F and PBDTT-PPzBT-O were synthesized by copolymerizing electron-rich 5,8-dialkylthienyl substituted benzo[1,2-b:4,5- $\left.b^{\prime}\right]$ dithiophene (BDTT) and two electron-deficient phenanthro[4,5-abc]phenazine (PPz) and benzo[c] $[1,2,5]$ thiadiazole (BT) units. By changing the substituents at the 5,6-positions of BT, the optoelectronic properties of the terpolymers could be rationally adjusted for application as donor materials in polymer solar cells (PSCs). As a result, these terpolymers exhibited different light absorption properties, HOMO energy levels and hole mobilities, which contributed to the optimization of short-circuit current $\left(J_{s c}\right)$, opencircuit voltage $\left(V_{O c}\right)$ and fill factor (FF) properties, respectively. Interestingly, a maximum power conversion efficiency (PCE) of $6.3 \%$ was obtained with an $V_{\text {oc }}$ of $0.75 \mathrm{~V}$, a J $J_{\text {sc }}$ of $13.0 \mathrm{~mA} \mathrm{~cm}^{-2}$ and a $\mathrm{FF}$ of $64.8 \%$ in the PBDTT-PPzBT-O based PSCs using [6,6]-phenyl- $C_{71}$-butyric acid methyl ester $\left(\mathrm{PC}_{71} \mathrm{BM}\right)$ as an acceptor, while PBDTT-PPzBT-H and PBDTT-PPzBT-F based devices also demonstrated a PCE of more than 4.5\%. To the best of our knowledge, these are the highest recorded maximum PCE, $J_{\mathrm{sc}}$ and FF values obtained to date compared with previously reported phenazine copolymeric derivatives in BHJ-PSCs. This study illustrates the potential of these random conjugated terpolymers as promising donor materials in the application of PSCs.
\end{abstract}

Received 20th March 2015, Accepted 12th May 2015

DOI: $10.1039 / c 5 t c 00785 b$

www.rsc.org/MaterialsC interactions. ${ }^{4-10}$ Recently, a major breakthrough was made in enhancing power conversion efficiencies (PCE) due to the development of novel conjugated polymers. ${ }^{11-14}$ For example, a record-setting PCE value of up to $10.8 \%$ had been harvested in PSCs. $^{11}$

Optimized photovoltaic properties in a polymer donor material can offer a high short-circuit current density $\left(J_{\mathrm{sc}}\right)$, a high open-circuit voltage $\left(V_{\mathrm{oc}}\right)$, and a high fill factor $(\mathrm{FF})$ and result in a high PCE from PSCs. ${ }^{15-17}$ Unfortunately, most D-A alternating polymers are unable to obtain high PCE due to the imbalance between $J_{\mathrm{sc}}$ and $V_{\mathrm{oc}}{ }^{18,19} \mathrm{In}$ this regard, terpolymers composed of three different units in the polymer backbone are promising candidates as they can exhibit the synergetic effects of each unit by tuning the composition ratio, thus demonstrating broad absorption abilities, deep highest occupied molecular orbital (HOMO) energy levels, high charge mobilities and good solubilities. ${ }^{20-29}$ For instance, Jo et al. obtained a class of random conjugated terpolymers by tuning the composition ratio of diketopyrrolopyrrole (DPP) and isoindigo as co-electron accepting units in D-A-type polymers. ${ }^{21}$ This type of a terpolymer provides a suitable balance between light absorption and photon flux from the solar spectrum, and a PCE of $6.04 \%$ with a $V_{\text {oc }}$ of $0.77 \mathrm{~V}$, 
$J_{\mathrm{sc}}$ of $13.52 \mathrm{~mA} \mathrm{~cm}^{-2}$ and $\mathrm{FF}$ of $58.0 \%$ was obtained from these terpolymer based BHJ-PSCs. Wei et al. exhibited another class of terpolymers with a D-A-type alternating backbone of benzodithiophene (BDT)-benzoxadiazole-DPP by tuning the composition ratio of two co-electron accepting units. ${ }^{22}$ Similar to other analogues, ${ }^{23-25}$ a maximum PCE of $6.8 \%$ with a $V_{\text {oc }}$ of $0.73 \mathrm{~V}, J_{\mathrm{sc}}$ of $17.0 \mathrm{~mA} \mathrm{~cm}^{-2}$ and $\mathrm{FF}$ of $55.0 \%$ was achieved in the polymers based on PSCs. Recently, phenazine derivatives as acceptor units have shown promising properties for application in PSCs, due to their relatively large $\pi$-conjugated system with a planar structure that is favorable for strong intermolecular $\pi-\pi$ stacking and its selfassembled characteristics, thus benefiting charge transportation and absorption of longer wavelengths. ${ }^{30-33}$ For instance, Jen et al. reported a polymer of PIDT-phanQ with an alternating D-A backbone of indacenodithiophene (IDT) and phenanthrene-quinoxaline (phanQ), a maximum PCE of $6.24 \%$ with a hole mobility $\left(\mu_{\mathrm{h}}\right)$ of $0.024 \mathrm{~cm}^{2} \mathrm{~V}^{-1} \mathrm{~s}^{-1}$ was obtained in PIDT-phanQ based PSCs. ${ }^{30}$ Our group also reported a polymer of PTTPPz-BDTT with the phenanthro[4,5- $a b c]$ phenazine $(\mathrm{PPz})$ acceptor unit and a maximum PCE of $4.87 \%$ with a FF of $62.5 \% .^{31}$

In addition, another type of polymer with 7,8-dialkylthienyl benzo[1,2- $\left.b: 4,5-b^{\prime}\right]$ dithiophene (BDTT) units was utilized as the donor material for application in PSCs. Due to its relatively large and planar conjugated structure, it was favorable for $\pi-\pi$ stacking and provided an improvement for carrier transportation and light absorption. ${ }^{34-36}$ For example, a maximum PCE of $9.48 \%$ with a $J_{\mathrm{sc}}$ of $17.46 \mathrm{~mA} \mathrm{~cm} \mathrm{~cm}^{-2}$ and $\mu_{\mathrm{h}}$ of $0.01 \mathrm{~cm}^{2} \mathrm{~V}^{-1} \mathrm{~s}^{-1}$ was obtained from PBDT-TS1 based PSCs by the Hou group. ${ }^{34}$ Furthermore, some benzo[c] $[1,2,5]$ thiadiazole (BT) derivatives as acceptor units have been widely used with D-A-type polymers for application in PSCs due to their strong electron-accepting abilities and properties deriving from substitution at the 5,6-positions. ${ }^{37-42}$ Woo et al. reported a polymer of PTBT14 with a 5,6-dialkoxy substituted BT-O as the acceptor unit, and a maximum PCE of $5.56 \%$ with a $\mu_{\mathrm{h}}$ of $0.02 \mathrm{~cm}^{2} \mathrm{~V}^{-1} \mathrm{~s}^{-1}$ was achieved in this PTBT14 based PSC. ${ }^{39}$ Watkins et al. reported a polymer of BFS4 with a 5,6-difluoro substituted BT-F as the acceptor unit, and a maximum PCE of $7.80 \%$ with a $V_{\mathrm{oc}}$ of $0.90 \mathrm{~V}, J_{\mathrm{sc}}$ of $14.2 \mathrm{~mA} \mathrm{~cm}{ }^{-2}$ and $\mathrm{FF}$ of $61.1 \%$ was obtained in this BFS4 based PSCs. ${ }^{41}$

Inspired by the aforementioned works, the synthesis and optical and electrochemical characterization of three novel D-A-type random conjugated terpolymers is reported herein with their photovoltaic performances in PSCs. Their molecular structures are shown in Scheme 1. It was expected that the construction of the BDTT-PPzBT backbone, and changes to the substituents at 5,6-positions of the BT unit, would enhance intramolecular ICT interactions and intermolecular $\pi-\pi$ stacking, thereby broadening the absorption spectrum, reducing the HOMO energy level, increasing the hole mobility and improving the photovoltaic properties of the resulting terpolymers. As expected, the PSC based on PBDTT-PPzBT-O/PC ${ }_{71} \mathrm{BM}$ exhibited the best performance with a PCE of $6.3 \%, V_{\mathrm{oc}}$ of $0.75 \mathrm{~V}, J_{\mathrm{sc}}$ of $13.0 \mathrm{~mA} \mathrm{~cm} \mathrm{~cm}^{-2}$ and $\mathrm{FF}$ of $64.8 \%$. This suggested that changing the substituents at the 5,6-positions of the BT unit provides a potential for application in organic solar cells. Future work will focus on tuning the energy levels of the terpolymers to achieve enhanced photovoltaic performances.

\section{Experimental section}

\subsection{Instrumentations and characterization}

Molecular weights were determined using a Waters GPC 2410 in tetrahydrofuran via a calibration curve employing polystyrene as the standard. Thermogravimetric analyses (TGA) were conducted under a dry nitrogen gas flow at a heating rate of $20{ }^{\circ} \mathrm{C} \min ^{-1}$ on a Perkin-Elmer TGA 7. Differential scan calorimetry (DSC) measurements were carried out with a Netzsch DSC-204 under nitrogen flow at heating and cooling rates of $10{ }^{\circ} \mathrm{C} \mathrm{min}{ }^{-1}$. UV-Vis absorption spectra were recorded on a HP-8453 UV-visible system. Cyclic voltammograms (CV) were carried out on a CHI660A electrochemical workstation with a three electrode electrochemical cell in a $0.1 \mathrm{M}$ tetrabutylammonium hexafluorophosphate $\left(\mathrm{TBAPF}_{6}\right)$ acetonitrile solution at a scan rate of $100 \mathrm{mV} \mathrm{s}^{-1}$ at room temperature under an argon atmosphere. In this three-electrode

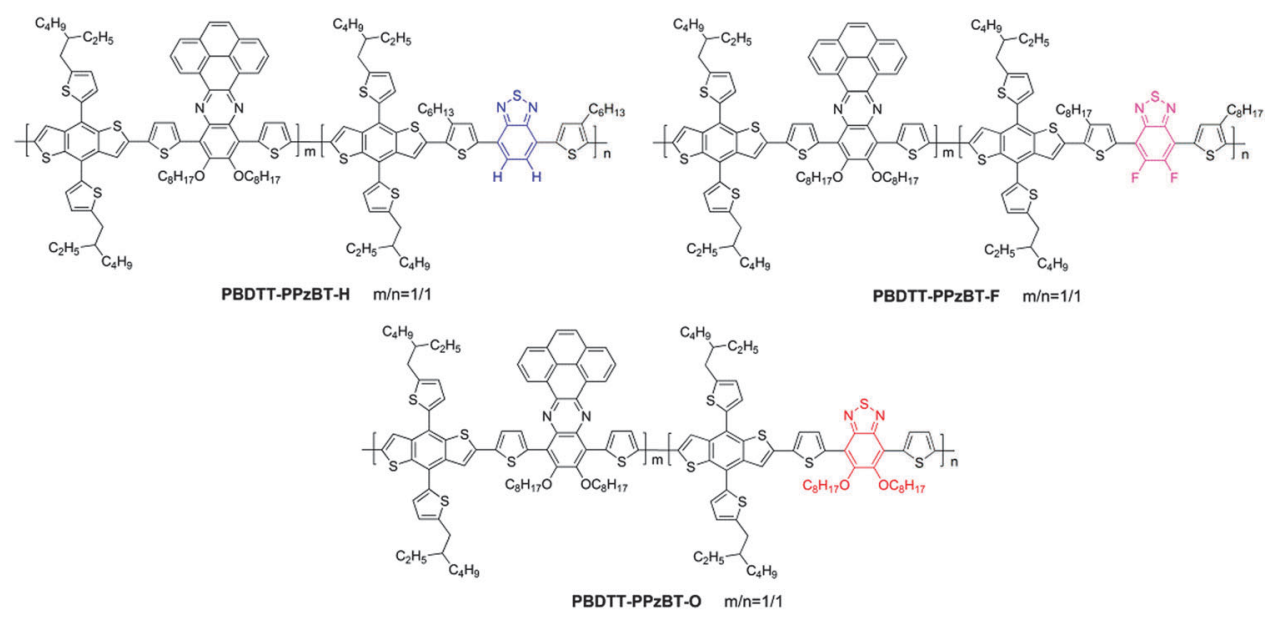

Scheme 1 Chemical structures of the terpolymers. 
cell, a platinum rod, a platinum wire and a $\mathrm{Ag} / \mathrm{AgCl}$ electrode were used as the working electrode, counter electrode and reference electrode, respectively, and was calibrated against the redox potential of ferrocene/ferrocenium $\left(\mathrm{Fc} / \mathrm{Fc}^{+}\right)$. Surface morphologies were recorded by atomic force microscopy (AFM) on a Veeco-134 DI multimode NS-3D apparatus in the tapping mode at room temperature under normal air conditions.

\subsection{Fabrication and characterization of polymer solar cells}

The PSCs were fabricated using indium tin oxide (ITO) glass as an anode, $\mathrm{Ca} / \mathrm{Al}$ as a cathode, and a blended film of the polymer/PCBM as a photosensitive layer. After a $30 \mathrm{~nm}$ buffer layer of poly(3,4-ethylenedioxythiophene) and polystyrene sulfonic acid (PEDOT:PSS) was spin-coated onto the pre-cleaned ITO substrate, the photosensitive layer was prepared by spin-coating a solution of the polymer/ $\mathrm{PC}_{71} \mathrm{BM}(1: 2, \mathrm{w} / \mathrm{w})$ in 1,2-dichlorobenzene (ODCB) on the PEDOT:PSS layer with a typical concentration of 15 (or 30) $\mathrm{mg} \mathrm{mL}^{-1}$, followed by annealing at $80{ }^{\circ} \mathrm{C}$ for 10 minutes to remove ODCB. Ca $(10 \mathrm{~nm})$ and $\mathrm{Al}(100 \mathrm{~nm})$ were successively deposited on the photosensitive layer in vacuum and used as top electrodes. The current-voltage $(I-V)$ characterization of the devices was carried out on a computer-controlled Keithley source measurement system. A solar simulator was used as the light source and the light intensity was monitored by a standard Si solar cell. The active area was $7 \times 10^{-2} \mathrm{~cm}^{2}$ for each cell. The thicknesses of the spun-cast films were recorded by a profilometer (Alpha-Step 200, Tencor Instruments). The external quantum efficiency (EQE) was measured with a Stanford Research Systems model SR830 DSP lock-in amplifier coupled with a WDG3 monochromator and a $150 \mathrm{~W}$ xenon lamp.

\subsection{Materials}

2,5-Bis(trimethyltin)-7,8-bis(5-(2-ethylhexyl)thiophen-2-yl)benzo[1,2-b:4,5- $\left.b^{\prime}\right]$ dithiophene (M1), 4,7-bis(5-bromo-4-hexylthiophen2-yl)benzo[c][1,2,5]thiadiazole (M3), 4,7-bis(5-bromo-4-octylthiophen2-yl)-5,6-difluorobenzo[ $c][1,2,5]$ thiadiazole (M4) and 4,7-bis(5bromothiophen-2-yl)-5,6-dioctyloxybenzo[c][1,2,5]thiadiazole (M5) were purchased directly from Suna Tech Inc. The other reagents and chemicals were purchased from commercial sources (Aldrich, TCI) and used without further purification unless stated otherwise. The monomer 10,13-bis(5-bromothiophen-2-yl)-11,12-bis(octyloxy)phenanthro[4,5-abc]phenazine (M2) was prepared according to literature procedures. ${ }^{31}$ The detailed syntheses of these terpolymers are presented in the following procedures.

\subsection{Synthesis of terpolymers}

2.4.1. Synthesis of terpolymer PBDTT-PPzBT-H. In a dry $25 \mathrm{~mL}$ flask, tris(dibenzylideneacetone)dipalladium(0) $\left(\mathrm{Pd}_{2}(\mathrm{dba})_{3}\right.$, $5.0 \mathrm{mg})$ and tri $(o$-tolyl $)$ phosphine $\left(\mathrm{P}(o-\mathrm{Tol})_{3}, 10.0 \mathrm{mg}\right)$ were added to a solution of M1 (118 mg, $0.13 \mathrm{mmol}), \mathrm{M} 2(57 \mathrm{mg}, 0.065 \mathrm{mmol})$ and M3 (41 mg, $0.065 \mathrm{mmol}$ ) in $8 \mathrm{~mL}$ of degassed toluene under a nitrogen atmosphere and stirred vigorously at $100{ }^{\circ} \mathrm{C}$ for $0.5 \mathrm{~h}$ until the reaction system became viscous. After cooling to room temperature, the mixture was poured into acetone $(100 \mathrm{~mL})$ and precipitation occurred. The solids were collected by filtration and Soxhlet-extracted successively with hexane, acetone and diethyl ether each for $12 \mathrm{~h}$. Then, the solvent was changed to chloroform $\left(\mathrm{CHCl}_{3}\right)$. The remaining solid was dissolved with ODCB $(100 \mathrm{~mL})$ and precipitated with methanol to get a dark solid (127 mg, 83.6\%). Anal. calcd for $\mathrm{C}_{140} \mathrm{H}_{158} \mathrm{~N}_{4} \mathrm{O}_{2} \mathrm{~S}_{13}$ : C, 71.69; H, 6.79; N, 2.39; S, 17.77. Found: C, 71.00; H, 7.30; N, $2.38 ; \mathrm{S}, 18.67$.

2.4.2. Synthesis of terpolymer PBDTT-PPzBT-F. PBDTTPPzBT-F was prepared according to the synthetic procedure of PBDTT-PPzBT-H by the reaction with M1 (118 $\mathrm{mg}, 0.13 \mathrm{mmol})$, M2 (57 mg, $0.065 \mathrm{mmol}$ ), M4 (47 mg, $0.065 \mathrm{mmol}), \mathrm{Pd}_{2}(\mathrm{dba})_{3}$ $(5.0 \mathrm{mg})$ and $\mathrm{P}(o-\mathrm{Tol})_{3}(10.0 \mathrm{mg})$ in dry toluene $(8 \mathrm{~mL})$. The reaction was stirred for $0.5 \mathrm{~h}$ until the reaction system became viscous and the terpolymer was collected as a dark solid $(129 \mathrm{mg}$, 81.6\%). Anal. calcd for $\mathrm{C}_{144} \mathrm{H}_{164} \mathrm{~F}_{2} \mathrm{~N}_{4} \mathrm{O}_{2} \mathrm{~S}_{13}$ : C, 70.95; H, 6.87; N, 2.30; S, 17.10. Found: C, 70.34; H, 7.69; N, 2.29; S, 18.31.

2.4.3. Synthesis of terpolymer PBDTT-PPzBT-O. PBDTTPPzBT-O was prepared according to the synthetic procedure of PBDTT-PPzBT-H by the reaction with M1 (117 $\mathrm{mg}, 0.13 \mathrm{mmol})$, M2 (57 mg, $0.065 \mathrm{mmol}$ ), M5 (46 mg, $0.065 \mathrm{mmol}), \mathrm{Pd}_{2}(\mathrm{dba})_{3}$ $(5.0 \mathrm{mg})$ and $\mathrm{P}(o-\mathrm{Tol})_{3}(10.0 \mathrm{mg})$ in dry toluene $(8 \mathrm{~mL})$. The reaction was stirred for $0.5 \mathrm{~h}$ until the reaction system became viscous and the terpolymer was collected as a dark solid (141 mg, 89.2\%). Anal. calcd for $\mathrm{C}_{144} \mathrm{H}_{166} \mathrm{~N}_{4} \mathrm{O}_{4} \mathrm{~S}_{13}$ : C, 71.07; H, 6.87; N, $2.30 ;$ S, 17.07. Found: C, 71.08; H, 7.76; N, 2.33; S, 18.43 .

\section{Results and discussion}

\subsection{Synthesis and thermal properties}

The synthetic routes for the three terpolymers of PBDTTPPzBT-H, PBDTT-PPzBT-F and PBDTT-PPzBT-O are outlined in Scheme 2. All the terpolymers were synthesized at $100{ }^{\circ} \mathrm{C}$ for $0.5 \mathrm{~h}$ by the palladium-catalyzed Stille-coupling polymerization in moderate yields. The actual molecular composition of these terpolymers was determined by elemental analysis. The results showed that the M1/M2/M3 (or M4 or M5) ratio was 2/1/1 in these terpolymers, and was consistent with the molar feed ratio of M1/M2/M3 (or M4 or M5). The molecular weight of these terpolymers were determined by GPC relative to polystyrene standards and the related data are listed in Table 1 . The average molecular weights $\left(M_{\mathrm{n}}\right)$ were observed to be $29 \mathrm{kDa}$ for PBDTTPPzBT-H, $45 \mathrm{kDa}$ for PBDTT-PPzBT-F, and $52 \mathrm{kDa}$ for PBDTTPPzBT-O. Although the molar mass of the synthesized terpolymers showed differences, all of these terpolymers exhibited similar solubility, and were only slightly soluble in most organic solvents, such as $\mathrm{CHCl}_{3}$, tetrahydrofuran (THF), chlorobenzene (CB) and ODCB under room temperature. The poor solubility of these terpolymers may be explained by the large planar polymer backbone that induces stronger intermolecular interactions. ${ }^{31-33}$

The recorded TGA curves of these random terpolymers are depicted in Fig. 1, and the corresponding data summarized in Table 1 . The thermal decomposition temperatures $\left(T_{\mathrm{d}}\right)$ are observed to be $350{ }^{\circ} \mathrm{C}$ for PBDTT-PPzBT-H, $363{ }^{\circ} \mathrm{C}$ for PBDTT-PPzBT-F and $341{ }^{\circ} \mathrm{C}$ for PBDTT-PPzBT-O at $5 \%$ weight loss. Compared to PBDTT-PPzBT-H, PBDTT-PPzBT-F exhibits a increased $T_{\mathrm{d}}$ value and PBDTT-PPzBT-O exhibits a decreased 


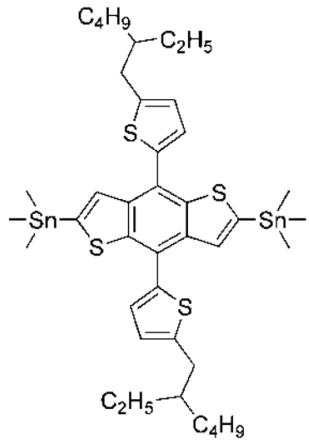

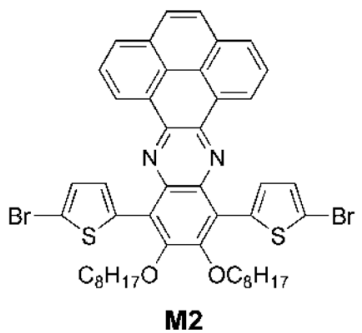

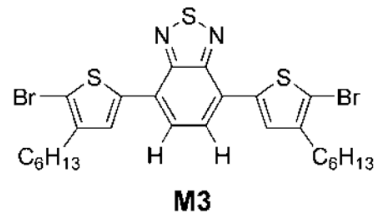<smiles>[CH]c1cc(-c2c(F)c(F)c3nsnc3c2-c2cc(C(F)F)c(Br)s2)sc1Br</smiles><smiles>CCOc1c(OC)c(-c2ccccc2)c2nsnc2c1-c1ccc(Br)s1</smiles>

$$
\begin{aligned}
& \mathbf{M} 1+\mathbf{M} 2+\mathbf{M} 3 \stackrel{\mathrm{P}(\mathrm{O}-\mathrm{toly})_{3}, \mathrm{Pd}_{2}(\mathrm{dba})_{3}}{\text { toluene }} \text { PBDTT-PPzBT-H } \mathrm{m} / \mathrm{n}=1 / 1 \\
& \mathbf{M} 1+\mathbf{M} 2+\mathbf{M} 4 \underset{\text { toluene }}{\stackrel{\mathrm{P}\left(\mathrm{o}-\text { toly }_{3} \mathrm{Pd}_{2}(\mathrm{dba})_{3}\right.}{\longrightarrow}} \quad \text { PBDTT-PPzBT-F } \quad \mathrm{m} / \mathrm{n}=1 / 1 \\
& \mathrm{M} 1+\mathrm{M} 2+\mathrm{M} 5 \frac{\mathrm{P}(\mathrm{O}-\mathrm{to} / \mathrm{y})_{3}, \mathrm{Pd}_{2}(\mathrm{dba})_{3}}{\text { toluene }} \quad \text { PBDTT-PPzBT-O } \mathrm{m} / \mathrm{n}=1 / 1
\end{aligned}
$$

Scheme 2 Synthetic routes of the terpolymers.

Table 1 Molecular weight and thermal properties of the terpolymers

\begin{tabular}{llcll}
\hline Terpolymers & $M_{\mathrm{n}}(\mathrm{kDa})$ & $M_{\mathrm{w}}(\mathrm{kDa})$ & PDI & $T_{\mathrm{d}}\left({ }^{\circ} \mathrm{C}\right)$ \\
\hline PBDTT-PPzBT-H & 29 & 90 & 3.10 & 350 \\
PBDTT-PPzBT-F & 45 & 110 & 2.24 & 363 \\
PBDTT-PPzBT-O & 52 & 131 & 2.52 & 341
\end{tabular}

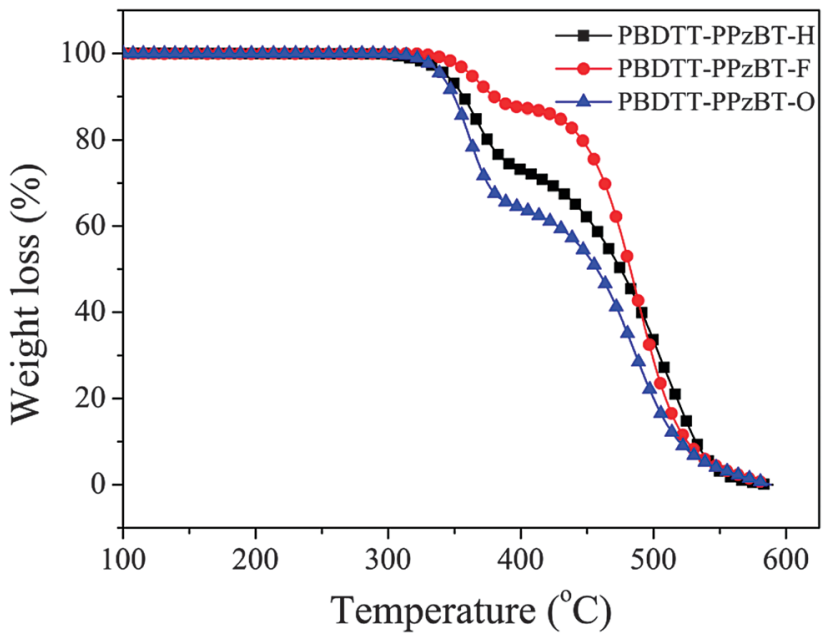

Fig. 1 TGA curves of the terpolymers.

$T_{\mathrm{d}}$ value, which means that two fluorine atoms (or two octyloxy groups) at the 5,6-positions of BT play a positive (or negative) role in increasing the thermal stability of its polymer. Moreover, no thermal transition points were observed from $50{ }^{\circ} \mathrm{C}$ to $180{ }^{\circ} \mathrm{C}$ in the DSC curves (Fig. S1, ESI $\dagger$ ), indicating that these terpolymers have amorphous characteristics. ${ }^{43}$

\subsection{Optical properties}

Fig. 2a and b show the normalized UV-Vis absorption spectra of these terpolymers in an ODCB solution with a concentration of $1 \times 10^{-5} \mathrm{M}$ and as a neat film. The detailed parameters are summarized in Table 2 . In solution, all the terpolymers exhibited a similar UV-Vis spectra with two absorption bands, among which the high-lying absorption band from 340 to $500 \mathrm{~nm}$ was assigned to the $\pi-\pi^{*}$ transition, and the other low-lying absorption band from 500 to $800 \mathrm{~nm}$ was attributed to a strong photoinduced ICT effect from the BDTT donor unit to the PPz and BT acceptor units. Interestingly, the three terpolymers showed similar absorption profiles with a slight shift in an ODCB solution (Fig. 2a). In general, the absorption spectra of polymers move to longer wavelength region by the introduction of electron donating units $(\mathrm{O}>\mathrm{H}>\mathrm{F})$; however, the appending two octyloxy groups at the 5,6-positions of BT cause the corresponding terpolymer PBDTT-PPzBT-O to blue-shift the absorption spectrum. Therefore, alkyloxy groups appended at the BT unit do not always broaden the absorption spectrum for its copolymers despite the fact that the alkyloxy group is a common electron-donating group. Similar reports were observed in other dioctyloxy side chain substituted D-A polymers. ${ }^{12}$ Compared with the absorption spectra in an ODCB solution, these terpolymers as neat films displayed an additional shoulder peak at 658, 602 and $596 \mathrm{~nm}$ in addition to the red-shifted 

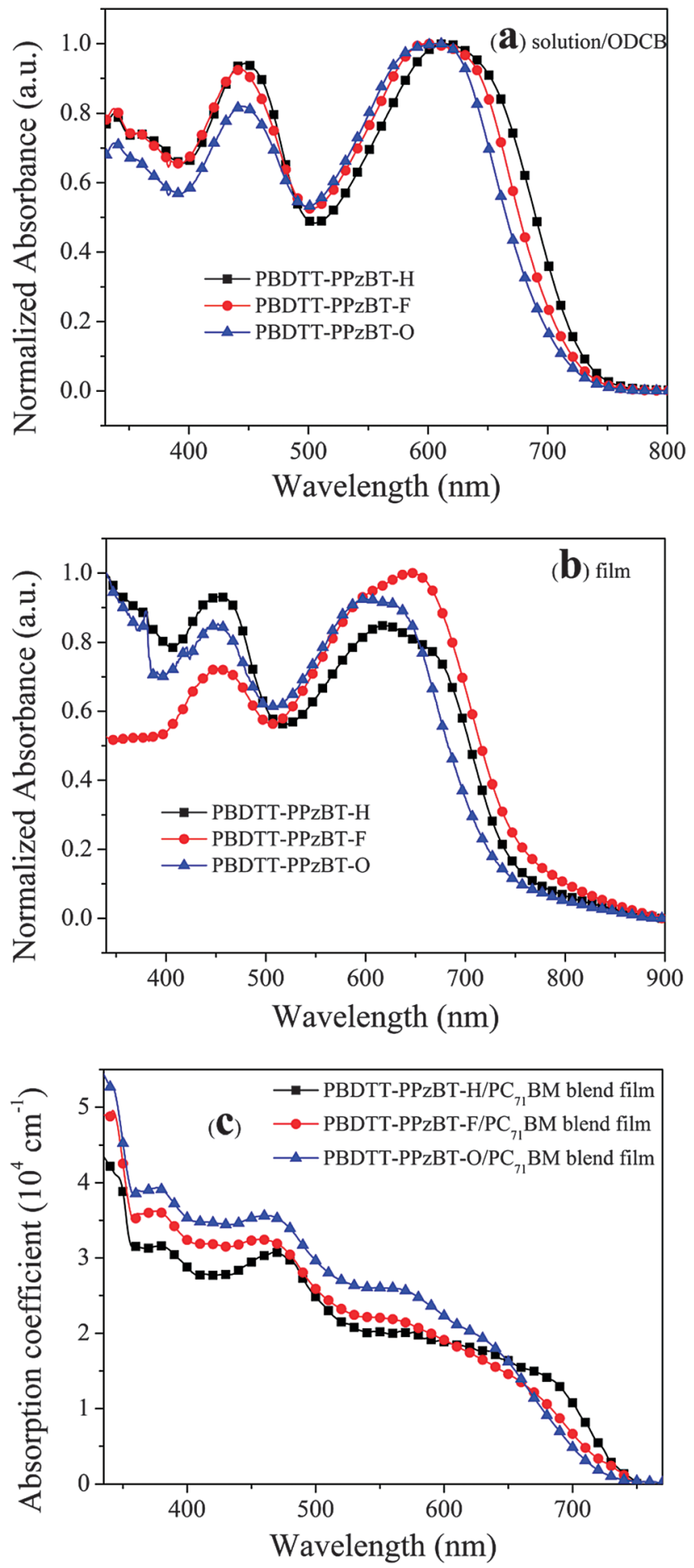

Fig. 2 UV-Vis absorption spectra of the terpolymers in solution (a), as thin films (b), and in their blended films with $\mathrm{PC}_{71} \mathrm{BM}$ at the optimized ratio (c).

high energy peak of 618, 649 and $626 \mathrm{~nm}$ for PBDTT-PPzBT-H, PBDTT-PPzBT-F and PBDTT-PPzBT-O (Fig. 2b), respectively, implying that all of these terpolymers possessed strong intermolecular packing potential in the solid state. Furthermore, PBDTT-PPzBT-F exhibited the largest red-shift absorption, about $45 \mathrm{~nm}$, which was ascribed to the fluorine atom substituent effect strengthening the intermolecular packing potential, ${ }^{44}$ while the other two terpolymers displayed some red-shifts as thin films. Based on the onset of the film absorption, the optical band gaps ( $E_{\mathrm{g}}^{\text {opt }}$ ) of PBDTT-PPzBT-H, PBDTT-PPzBT-F and PBDTT-PPzBT-O were calculated as 1.61, 1.56 and $1.63 \mathrm{eV}$, respectively. The extended absorption edge in the long wavelength region was attributed to the more aggregated configuration formed in the solid state. Fig. 2c shows the UV-Vis absorption spectra of the terpolymer/PC $\mathrm{P}_{71} \mathrm{BM}$ blended films at the optimized ratio of $1 / 2(\mathrm{w} / \mathrm{w})$. Increasing absorption intensities were observed in PBDTT-PPzBT-H, PBDTT-PPzBT-F and PBDTT-PPzBT-O blended films. This suggested that the two fluorine atoms or octyloxy groups, instead of the two hydrogen atoms at the 5,6-positions of BT, were able to enhance the UV-Vis absorption ability of the terpolymers.

\subsection{Electrochemical properties}

The electrochemical properties of the terpolymers were investigated through cyclic voltammetry (CV). Fig. 3 shows the recorded $\mathrm{CV}$ curves of the terpolymers using an $\mathrm{Ag} / \mathrm{AgCl}$ electrode as the reference and the redox potential of ferrocene/ ferrocenium $\left(\mathrm{Fc} / \mathrm{Fc}^{+}\right)$as the calibrated standard. The relevant data are summarized in Table 2. The observed onset oxidation potentials $\left(E_{\text {ox }}\right)$ for PBDTT-PPzBT-H, PBDTT-PPzBT-F and PBDTT-PPzBT-O were $0.80 \mathrm{~V}, 0.97$ and $0.95 \mathrm{~V}$, respectively. The HOMO energy levels of the terpolymers were calculated according to the empirical equation: ${ }^{45} E_{\mathrm{HOMO}}=-\left(E_{\mathrm{ox}}-0.45\right)-$ $4.8 \mathrm{eV}$. As a result, the HOMO energy levels $\left(E_{\mathrm{HOMO}}\right)$ were estimated at $-5.15 \mathrm{eV}$ for PBDTT-PPzBT-H, $-5.32 \mathrm{eV}$ for PBDTT-PPzBT-F and $-5.30 \mathrm{eV}$ for PBDTT-PPzBT-O. The LUMO energy levels ( $E_{\mathrm{LUMO}}$ ) levels of the terpolymers were calculated by " $E_{\mathrm{LUMO}}=E_{\mathrm{HOMO}}+E_{\mathrm{g}}^{\mathrm{opt}}$ ". Compared to PBDTTPPzBT-H, PBDTT-PPzBT-F and PBDTT-PPzBT-O presented a significant decrease in the $E_{\text {Hомо }}$ levels due to hydrogen bonding effects. ${ }^{39-41}$ It is known that the $V_{\text {oc }}$ of BHJ-PSCs is directly proportional to the offset between the HOMO level of the donor and LUMO level of the acceptor. Thus, it could be concluded that the substitution of fluorine atoms or alkoxy groups improves the $V_{\text {oc }}$.

The HOMO and LUMO distributions of polymers were calculated by the density functional theory (DFT) (B3LYP; 6-31G*) method. As shown in Fig. 4, these terpolymers showed similar electron-state-density distributions of the HOMO, while two fluorine atoms or two octyloxyl groups at the 5,6-positions of BT instead of two hydrogen atoms slightly decreased the HOMO of these terpolymers and is consistent with CV measurements. It is worth noting that the electron density distributions of the LUMO are mostly located on the acceptor units of BT (or BT-F or BT-O), which means that the electron withdrawing ability of the BT (or BT-F or BT-O) unit is stronger than the $\mathrm{PPz}$ unit. The DFT calculations showed that BT (or BT-F or BT-O) is the major factor influencing the LUMO energy level of the terpolymers with the two electron-withdrawing units. These results are consistent with the DFT calculation results of BDTT-alt-DTPPz and BDTT-alt-DTBT copolymers (see Fig. S2, ESI $\dagger$ ). 
Table 2 Optical and electrochemical properties of the terpolymers

\begin{tabular}{|c|c|c|c|c|c|c|c|}
\hline Terpolymers & $\lambda_{\mathrm{abs}}{ }^{a} / \mathrm{nm}$ & $\lambda_{\mathrm{abs}}^{b} / \mathrm{nm}$ & $\lambda_{\text {onset }^{b}}^{b} / \mathrm{nm}$ & $E_{\mathrm{g}}^{\mathrm{opt}} / \mathrm{eV}$ & $E_{\mathrm{Ox}} / \mathrm{V}$ & $\mathrm{E}_{\text {номо }} / \mathrm{eV}$ & $E_{\mathrm{LUMO}}{ }^{c} / \mathrm{eV}$ \\
\hline PBDTT-PPzBT-F & 443,604 & $456,602,649$ & 795 & 1.56 & 0.97 & -5.32 & -3.76 \\
\hline
\end{tabular}

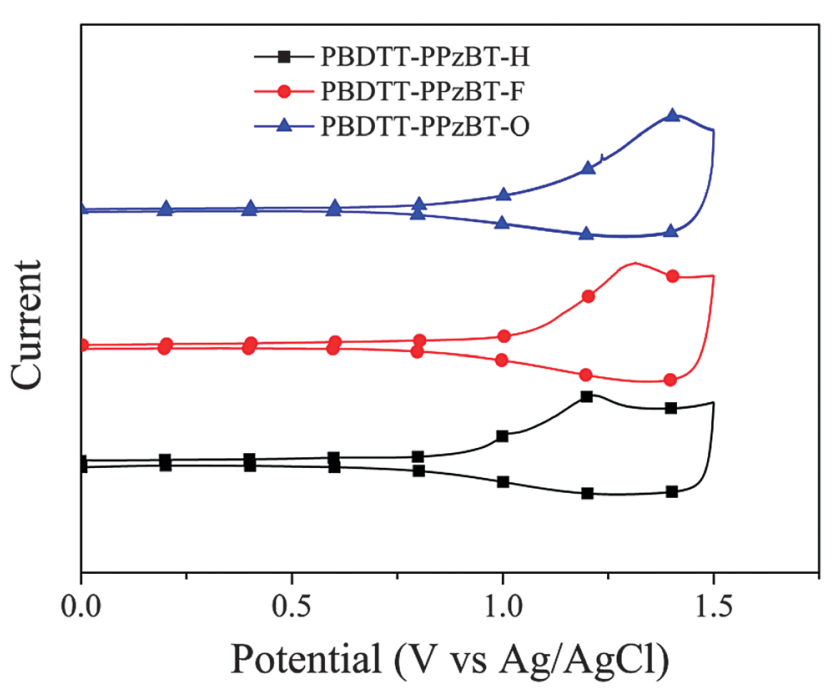

Fig. 3 Cyclic voltammogram curves for the terpolymers.

\subsection{Hole mobility}

To further understand the effect of chemical structures on the photovoltaic performance of terpolymers in PSC devices, the hole mobilities $\left(\mu_{\mathrm{h}}\right)$ of these photoactive layers were measured by the space charge limited current (SCLC) method in hole devices with a structure of ITO/PEDOT:PSS/terpolymer:PC ${ }_{71} \mathrm{BM} /$ $\mathrm{Au}$ at the optimized conditions. The SCLC could be estimated using the Mott-Gurney equation: $J=(9 / 8) \varepsilon_{0} \varepsilon_{\mathrm{r}} \mu_{\mathrm{h}}\left(V / L^{3}\right),{ }^{46}$ where $J$ is the current density, $\varepsilon_{\mathrm{r}}$ is the terpolymer dielectric constant, $\varepsilon_{0}$ is the free-space permittivity $\left(8.85 \times 10^{-12} \mathrm{~F} \mathrm{~m}^{-1}\right), L$ is blended film layer thickness and $V=V_{\text {appl }}-V_{\mathrm{bi}}$, where $V$ is the effective voltage, $V_{\mathrm{appl}}$ is the applied voltage, and $V_{\mathrm{bi}}$ is the built-in voltage that results from the work function difference between the anode and cathode. Fig. 5 displays the $J-V$ curves of these hole devices containing terpolymer/ $\mathrm{PC}_{71} \mathrm{BM}$ active layers. As summarized in Table 3, the hole mobilities of PBDTT-PPzBT-H, PBDTT-PPzBT-F and PBDTT-PPzBT-O were calculated as $4.64 \times 10^{-5}, 8.61 \times 10^{-5}$ and $3.75 \times 10^{-4} \mathrm{~cm}^{2} \mathrm{~V}^{-1} \mathrm{~s}^{-1}$, respectively, in the hole terpolymer/ $\mathrm{PC}_{71} \mathrm{BM}$-based devices. Compared with the previously reported binary polymer PTTPz-BDTT based on PPz and BDTT units, ${ }^{31}$ significantly increased mobility was achieved for PBDTT-PPzBT-O. This suggested that incorporating BT with two octyloxy substituents into a terpolymer facilitated increased hole mobility, which could be ascribed to enhanced inter-chain $\pi-\pi$ interactions. ${ }^{39}$ Obviously, high hole mobility for PBDTT-PPzBT-O was responsible for the high FF level and PCE in these devices.

\subsection{Photovoltaic properties}

To investigate the photovoltaic properties of the terpolymers, $\mathrm{BHJ}$-PSCs were also fabricated with a general device structure of ITO/PEDOT:PSS/active layer/Ca/Al. The active layer (D/A,

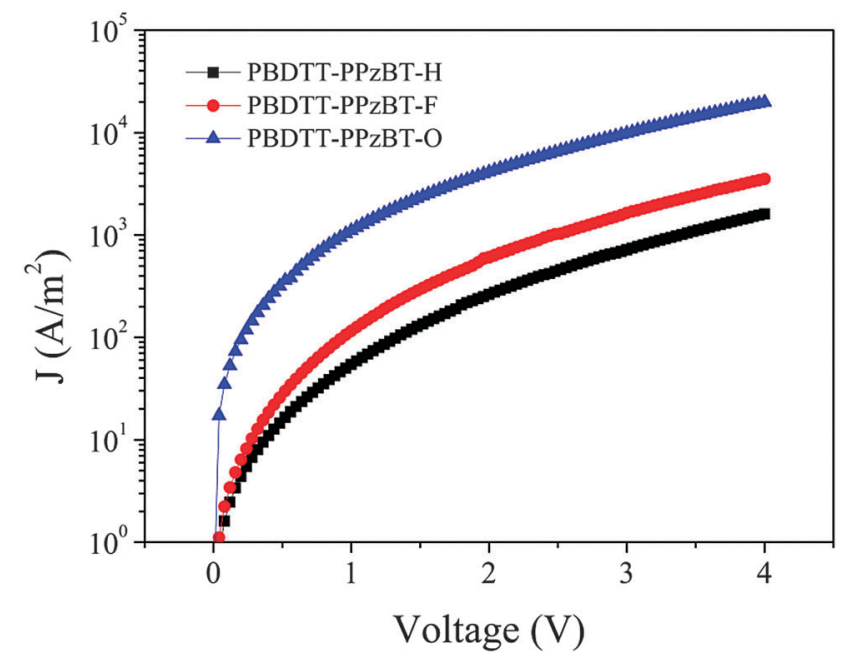

Fig. $5 \mathrm{~J}-V$ curves of the optimized hole-only terpolymer/PC ${ }_{71} \mathrm{BM}$ devices.

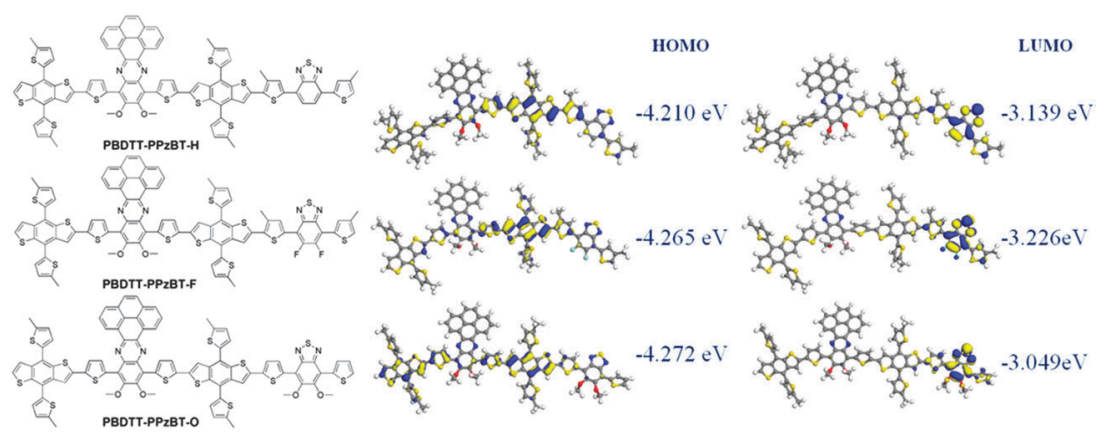

Fig. $4 \mathrm{HOMO}$ and LUMO distribution mode of terpolymers obtained using DFT calculations at the B3LYP/6-31G* level. 
Table 3 Photovoltaic properties of the terpolymer/PC ${ }_{71} \mathrm{BM}$-based PSCs at optimized conditions under illumination of AM 1.5G, $100 \mathrm{~mW} \mathrm{~cm}{ }^{-2}$

\begin{tabular}{|c|c|c|c|c|c|}
\hline Polymer/PC ${ }_{71} \mathrm{BM}$ & $\begin{array}{l}J_{\mathrm{sc}} l \\
\mathrm{~mA} \mathrm{~cm}\end{array}$ & $V_{\mathrm{oc}} / \mathrm{V}$ & $\mathrm{FF} / \%$ & $\mathrm{PCE}_{\max } / \%$ & $\begin{array}{l}\text { Hole mobility } \\
\mathrm{cm}^{2} \mathrm{~V}^{-1} \mathrm{~s}^{-1}\end{array}$ \\
\hline PBDTT-PPzBT-H & 12.4 & 0.67 & 55.0 & 4.5 & $4.64 \times 10^{-5}$ \\
\hline PBDTT-PPzBT-F & 12.5 & 0.74 & 59.6 & 5.5 & $8.61 \times 10^{-5}$ \\
\hline PBDTT-PPzBT-O & 13.0 & 0.75 & 64.8 & 6.3 & $3.75 \times 10^{-4}$ \\
\hline
\end{tabular}

terpolymer/PC ${ }_{71} \mathrm{BM}$ ) was obtained from an ODCB solution at different concentrations ( $30 \mathrm{mg} \mathrm{mL}{ }^{-1}$ for PBDTT-PPzBT-H and PBDTT-PPzBT-F, $15 \mathrm{mg} \mathrm{mL} \mathrm{mL}^{-1}$ for PBDTT-PPzBT-O). It is known that the photovoltaic performances of PSCs are strongly affected by the parameters used during construction, including the $\mathrm{D} / \mathrm{A}$ ratio $(\mathrm{w} / \mathrm{w})$, annealing temperature and spin-coating rate. ${ }^{31}$ Optimized parameters were obtained and Fig. S3-S5 (ESI $\dagger$ ) depict the $J-V$ characteristics of terpolymer/ $\mathrm{PC}_{71} \mathrm{BM}$-based devices at different ratios, annealing temperatures and spin-coating rates, respectively. The resulting photovoltaic data is summarized in Tables $\mathrm{S} 1-\mathrm{S} 3$ (ESI $\dagger$ ). The terpolymer/PC ${ }_{71} \mathrm{BM}$-based PSCs have an optimized $\mathrm{D} / \mathrm{A}$ ratio of $1: 2$ and annealing temperature of $80{ }^{\circ} \mathrm{C}$, while different spin-coating rates were utilized for the devices (3000 rpm for PBDTTPPzBT-H, $2500 \mathrm{rpm}$ for PBDTT-PPzBT-F, $1000 \mathrm{rpm}$ for PBDTTPPzBT-O). As shown in Fig. 6, the devices exhibited typical $J-V$ characteristics under these optimized conditions. Device parameters, such as $J_{\mathrm{sc}}, V_{\mathrm{oc}}$, FF and PCE, were deduced from the $J-V$ characteristics and are summarized in Table 3. All three of the devices exhibited excellent photovoltaic properties, in which PCE was more than $4.5 \%$ and $J_{\mathrm{sc}}$ more than $12.0 \mathrm{~mA} \mathrm{~cm}{ }^{-2}$.

As shown in Fig. 6, the PBDTT-PPzBT-F/PC ${ }_{71} \mathrm{BM}$ and PBDTTPPzBT-O/PC ${ }_{71} \mathrm{BM}$-based devices exhibited better photovoltaic properties compared with the PBDTT-PPzBT-H/PC ${ }_{71}$ BM-based device, which could be attributed to the two fluorine atoms and octyloxy units substituting BT in the terpolymers, causing significant increases in the $V_{\text {oc }}$ (from $0.67 \mathrm{~V}$ to $0.74 \mathrm{~V}$ and $0.75 \mathrm{~V}$ ) and $\mathrm{FF}$ (from $55.0 \%$ to $59.6 \%$ and $64.8 \%$ ) at their PSCs. Furthermore, compared with the PBDTT-PPzBT-F/PC ${ }_{71} \mathrm{BM}$-based device,

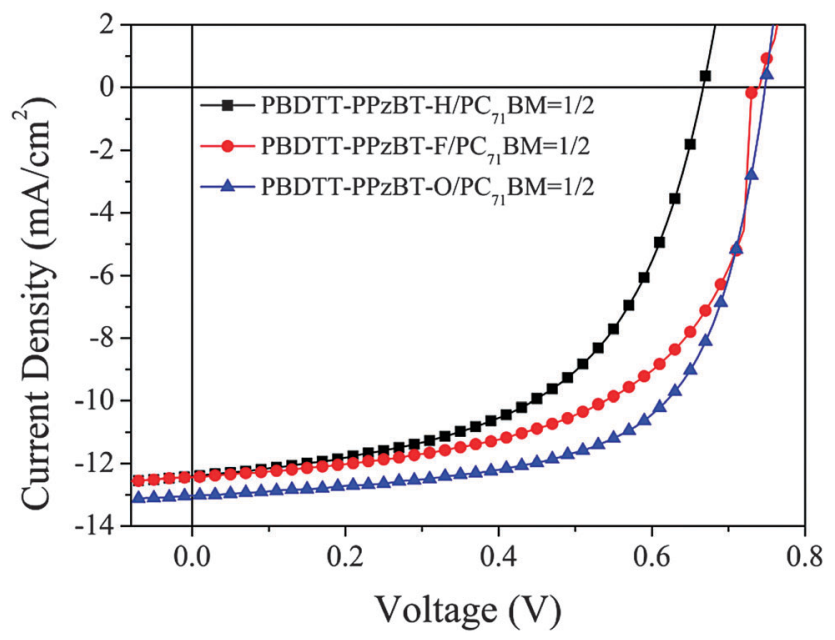

Fig. $6 J-V$ curves of the terpolymer/PC ${ }_{71} B M$-based $P S C s$ at optimized conditions under illumination of $A M 1.5 \mathrm{G}, 100 \mathrm{~mW} \mathrm{~cm} \mathrm{~cm}^{-2}$. the PBDTT-PPzBT-O/PC ${ }_{71} \mathrm{BM}$-based device showed a significant increase in the $\mathrm{FF}$ from $59.6 \%$ to $64.8 \%$. The high $\mathrm{FF}$ value was responsible for the high hole mobility of the PBDTT-PPzBT-O based hole devices. Notably, compared to the previous alternating M1-alt-M2 polymer PTTPPz-BDTT (PCE of $4.86 \%$ with a $V_{\mathrm{oc}}$ of $0.70 \mathrm{~V}, J_{\mathrm{sc}}$ of $11.1 \mathrm{~mA} \mathrm{~cm}{ }^{-2}$ and FF of $\left.62.5 \%\right),{ }^{31}$ PBDTT-PPzBT-F and PBDTT-PPzBT-O exhibited higher $V_{\mathrm{oc}}, J_{\mathrm{sc}}$ and PCE values, which was attributed the introduction of the second acceptor units of M4 or M5 in the terpolymer systems, and both reduced the HOMO levels and promoted the photonic response of these terpolymers. As a result, a maximal PCE of up to $6.3 \%$ was obtained with a $V_{\mathrm{oc}}$ of $0.75 \mathrm{~V}, J_{\mathrm{sc}}$ of $13.0 \mathrm{~mA} \mathrm{~cm} \mathrm{~cm}^{-2}$ and $\mathrm{FF}$ of $64.8 \%$ in the PBDTT-PPzBT-O/PC ${ }_{71} \mathrm{BM}$-based device. To the best of our knowledge, these are the highest PCE, $J_{\mathrm{sc}}$ and FF values recorded to date for BHJ-PSCs (compared with previously reported phenazine copolymeric derivatives), ${ }^{30-33}$ and can be attributed to the introduction of the two octyloxy substituents on BT.

In order to understand why the PBDTT-PPzBT-O/PC ${ }_{71} \mathrm{BM}$ based devices displayed the highest PCE values, the external quantum efficiency (EQE) curves of devices under the optimized conditions were also measured. As depicted in Fig. 7, all of the terpolymers/ $\mathrm{PC}_{71} \mathrm{BM}$-based devices demonstrated a very efficient photoresponse in the broad range from 310 to $750 \mathrm{~nm}$, corresponding to a high EQE of over $40 \%$ obtained in the broad range from 340 to $670 \mathrm{~nm}$ for all three devices. Maximum EQEs were obtained in the terpolymers/ $\mathrm{PC}_{71} \mathrm{BM}$-based devices of $72 \%$ at $424 \mathrm{~nm}$ for PBDTT-PPzBT-H, 79\% at $411 \mathrm{~nm}$ for PBDTTPPzBT-F and $83 \%$ at $400 \mathrm{~nm}$ for PBDTT-PPzBT-O. Obviously, the PBDTT-PPzBT-O based device showed a higher EQE in the range from 350 to $650 \mathrm{~nm}$ as compared to the PBDTT-PPzBT-H and PBDTT-PPzBT-F based devices. Higher EQE values and more intense absorptions (see Fig. 2c) powerfully facilitated the PBDTT-PPzBT-O based devices in providing higher $J_{\mathrm{sc}}$ values. Moreover, in accordance with the EQE curves and the solar irradiation spectrum, the integral $J_{\mathrm{sc}}$ values were determined to be 12.0, 12.2 and $12.6 \mathrm{~mA} \mathrm{~cm}^{-2}$ for PBDTT-PPzBT-H, PBDTTPPzBT-F and PBDTT-PPzBT-O based devices, respectively,

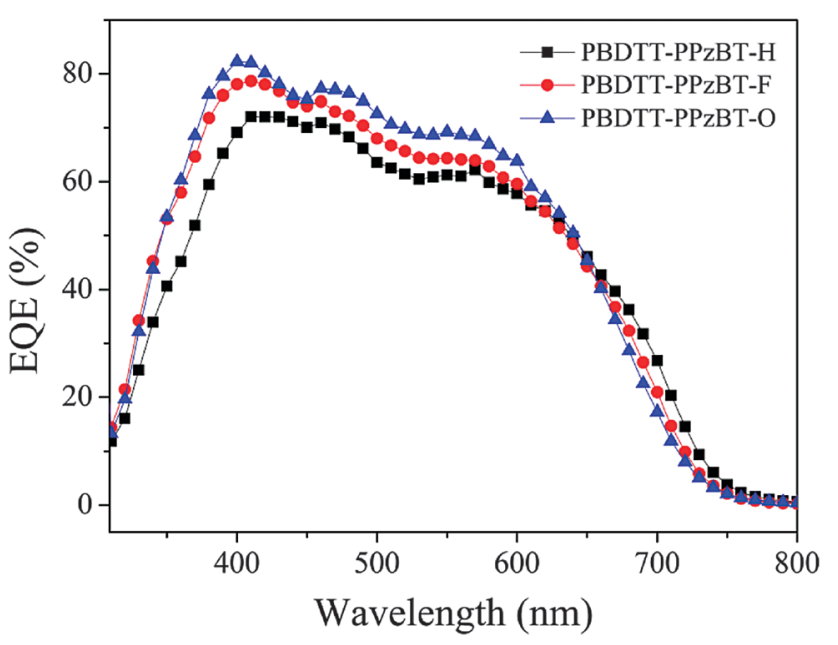

Fig. 7 EQE curves of the terpolymer/PC ${ }_{71} B M$-based devices. 
which were in agreement with the measured $J_{\mathrm{sc}}$ values within a $4 \%$ error margin.

\subsection{Film morphology}

The morphology of the blended films under the optimized conditions could also explain the enhanced photovoltaic performance of the PBDTT-PPzBT-O based device. Fig. 8 displays the surface morphology of the terpolymers $/ \mathrm{PC}_{71} \mathrm{BM}$ blended films as recorded by atomic force microscopy (AFM) in a surface area of $5 \times 5 \mu^{2}$ using the tapping mode. The root mean square (RMS) roughness from the height images were determined as 1.30, 1.26 and $0.91 \mathrm{~nm}$ for the PBDTT-PPzBT-H, PBDTT-PPzBT-F and PBDTT-PPzBT-O based blended films, respectively. A smoother surface was observed for the PBDTT-PPzBT-O based blended film. Therefore, higher $J_{\mathrm{sc}}$ and PCE values for the PBDTT-PPzBT-O based device were ascribed to more efficient charge transport properties and smoother morphologies, in addition to better absorption properties, which provided a $\mathrm{FF}$ of $64.8 \%$ and better device performances with a PCE of up to $6.3 \%$.

\subsection{XRD analysis}

The XRD patterns of these terpolymers were carried out in order to investigate the molecular interactions in detail and are shown in Fig. 9. It is observed that PBDTT-PPzBT-H, PBDTT-PPzBT-F and PBDTT-PPzBT-O show a strong peak at $2 \theta=22.1^{\circ}, 2 \theta=22.9^{\circ}$

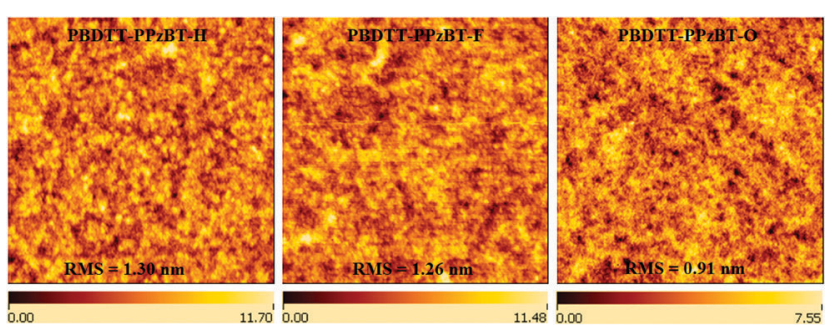

Fig. 8 AFM height images of the terpolymer/PC $C_{71} B M$ blend films under optimized conditions.

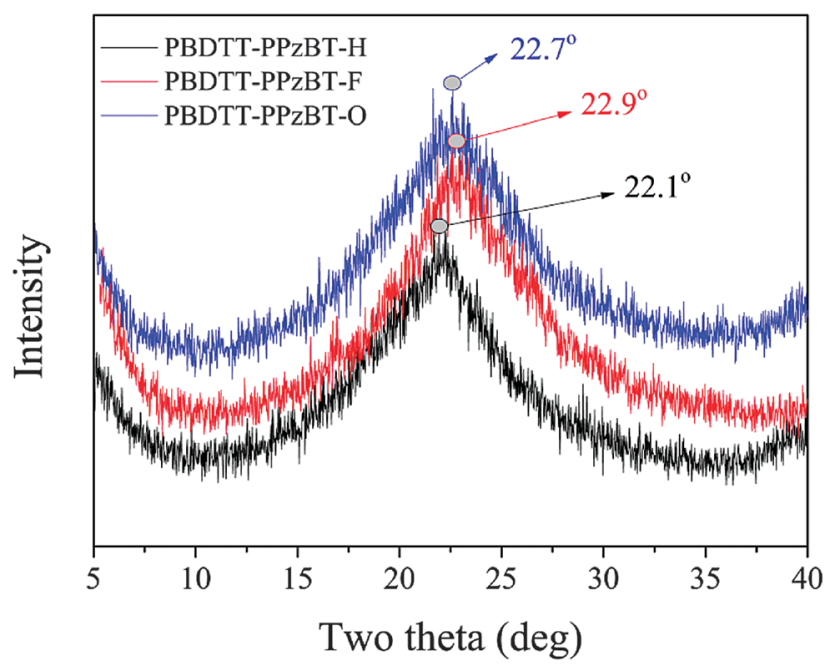

Fig. 9 XRD spectra of the terpolymers thin films. and $2 \theta=22.7^{\circ}$, respectively, indicating that all polymers have effective $\pi-\pi$ stacking due to the great aggregation of PPz unit. $^{31}$ Compared with PBDTT-PPzBT-H, PBDTT-PPzBT-F and PBDTTPPzBT-O exhibited a increasing $2 \theta$ peak. This implies that incorporating fluorine atom or octyloxy side chain substituents at the 5,6-positions of BT would enhance intermolecular $\pi-\pi$ stacking. It is consistent with the results of both AFM and charge carrier mobility measurements. The abovementioned investigations show that the large planar PPz unit and the 5,6-difluorine or 5,6-dioctyloxy substituted BT unit provides effective $\pi-\pi$ stacking of polymers, which is favorable for exciton dissociation and charge transportation, offering largely higher $J_{\mathrm{sc}}$ and $\mathrm{FF}$ for the resulting devices. ${ }^{31,40}$

\section{Conclusions}

In this study, three novel $\mathrm{A}_{1}-\mathrm{D}-\mathrm{A}_{2}$ terpolymers of PBDTT-PPzBT-H, PBDTT-PPzBT-F and PBDTT-PPzBT-O were synthesized by copolymerizing electron-rich BDTT and two electron-deficient PPz and BT units. By changing the substituents at the 5,6-positions of BT, the photophysical, electrochemical and charge transport properties of the terpolymers could be rationally adjusted. A maximum PCE of $6.3 \%$ with a $V_{\mathrm{oc}}$ of $0.75 \mathrm{~V}, \mathrm{Jsc}_{\mathrm{sc}}$ of $13.0 \mathrm{~mA} \mathrm{~cm} \mathrm{~cm}^{-2}$ and $\mathrm{FF}$ of $64.8 \%$ was exhibited in the PBDTT-PPzBT-O/PC ${ }_{71} \mathrm{BM}$ based PSCs. The other terpolymer based devices also demonstrated PCEs of more than $4.5 \%$. To the best of our knowledge, these are the highest recorded PCE, $J_{\mathrm{sc}}$ and FF values reported to date in BHJ-PSCs as compared with previously reported phenazine copolymeric derivatives. This study suggests that random conjugated terpolymers could be promising donor materials for application in PSCs.

\section{Acknowledgements}

This study was supported by the Major Program for cultivation of the National Natural Science Foundation of China (91233112), the National Natural Science Foundation of China (51273168, 21172187 and 21202139), the Ministry of Science and Technology of China (2010DFA52310), the Innovation Group and Xiangtan Joint Project of Hunan Natural Science Foundation (12JJ7002 and 12JJ8001), the key project of Hunan Provincial Education Department (13A102, 12B123), and the Postgraduate Science Foundation for Innovation in Hunan Province (CX2012B249, CX2013B268).

\section{Notes and references}

1 Y. F. Li, Acc. Chem. Res., 2012, 45, 723.

2 K. R. Graham, C. Cabanetos, J. P. Jahnke, M. N. Idso, A. E. Labban, G. O. N. Ndjawa, T. Heumueller, K. Vandewal, A. Salleo, B. F. Chmelka, A. Amassian, P. M. Beaujuge and M. D. McGehee, J. Am. Chem. Soc., 2014, 136, 9608.

3 P. Bujak, I. K. Bajer, M. Zagorska, V. Maurel, I. Wielgus and A. Pron, Chem. Soc. Rev., 2013, 42, 8895.

4 J. X. Wang, M. J. Xiao, W. C. Chen, M. Qiu, Z. K. Du, W. G. Zhu, S. G. Wen, N. Wang and R. Q. Yang, Macromolecules, 2014, 47, 7823. 
5 C. H. Cui, W. Y. Wong and Y. F. Li, Energy Environ. Sci., 2014, 7, 2276.

6 D. F. Dang, W. C. Chen, R. Q. Yang, W. G. Zhu, W. Mammod and E. G. Wang, Chem. Commun., 2013, 49, 9335.

7 M. Wang, H. B. Wang, T. Yokoyama, X. F. Liu, Y. Huang, Y. Zhang, T. Q. Nguyen, S. Aramaki and G. C. Bazan, J. Am. Chem. Soc., 2014, 136, 12576.

8 W. Y. Su, Q. P. Fan, M. J. Xiao, J. H. Chen, P. Zhou, B. Liu, H. Tan, Y. Liu, R. Q. Yang and W. G. Zhu, Macromol. Chem. Phys., 2014, 215, 2075.

9 K. Li, Z. J. Li, K. Feng, X. P. Xu, L. Y. Wang and Q. Peng, J. Am. Chem. Soc., 2013, 135, 13549.

10 K. Wang, Y. Zhao, W. L. Tang, Z. G. Zhang, Q. Fu and Y. F. Li, Org. Electron., 2014, 15, 818.

11 Y. H. Liu, J. B. Zhao, Z. K. Li, C. Mu, W. Ma, H. W. Hu, K. Jiang, H. R. Lin, H. Ade and H. Yan, Nat. Commun., 2014, $5,5293$.

12 W. Y. Su, M. J. Xiao, Q. P. Fan, J. Zhong, J. H. Chen, D. F. Dang, J. W. Shi, W. J. Xiong, X. W. Duan, H. Tan, Y. Liu and W. G. Zhu, Org. Electron., 2015, 17, 129.

13 H. C. Chen, Y. H. Chen, C. C. Liu, Y. C. Chien, S. W. Chou and P. T. Chou, Chem. Mater., 2012, 24, 4766.

14 W. T. Li, S. Albrecht, L. Q. Yang, S. Roland, J. R. Tumbleston, T. McAfee, L. Yan, M. A. Kelly, H. Ade, D. Neher and W. You, J. Am. Chem. Soc., 2014, 136, 15566.

15 J. Warnan, C. Cabanetos, R. Bude, A. E. Labban, L. Li and P. M. Beaujuge, Chem. Mater., 2014, 26, 2829.

16 C. Cabanetos, A. E. Labban, J. A. Bartelt, J. D. Douglas, W. R. Mateker, J. M. J. Fréchet, M. D. McGehee and P. M. Beaujuge, J. Am. Chem. Soc., 2013, 135, 4656.

17 T. L. Nguyen, H. Choi, S. J. Ko, M. A. Uddin, B. Walker, S. Yum, J. E. Jeong, M. H. Yun, T. J. Shin, S. Hwang, J. Y. Kim and H. Y. Woo, Energy Environ. Sci., 2014, 7, 3040.

18 J. H. Chen, Q. G. Liao, G. Ye, D. He, X. Y. Du, W. G. Zhu, J. H. Liao, Z. Xiao and L. M. Ding, Macromol. Chem. Phys, 2013, 214, 2054.

19 J. Warnan, A. E. Labban, C. Cabanetos, E. T. Hoke, P. K. Shukla, C. Risko, J. L. Brédas, M. D. McGehee and P. M. Beaujuge, Chem. Mater., 2014, 26, 2299.

20 H. Tan, J. T. Yu, Y. F. Wang, J. H. Chen, Q. Tao, Y. Liu, J. Huang and W. G. Zhu, Org. Electron., 2013, 14, 1510.

21 J. W. Jung, F. Liu, T. P. Russell and W. H. Jo, Energy Environ. Sci., 2013, 6, 3301.

22 J. M. Jiang, H. C. Chen, H. K. Lin, C. M. Yu, S. C. Lan, C. M. Liu and K. H. Wei, Polym. Chem., 2013, 4, 5321.

23 J. Li, K. H. Ong, P. Sonar, S. L. Lim, G. M. Ng, H. K. Wong, H. S. Tan and Z. K. Chen, Polym. Chem., 2013, 4, 804.

24 W. J. Sun, Z. F. Ma, D. F. Dang, W. G. Zhu, M. R. Andersson, F. L. Zhang and E. G. Wang, J. Mater. Chem. A, 2013, 1, 11141.

25 M. Zhang, F. Wu, Z. C. Cao, T. P. Shen, H. J. Chen, X. L. Li and S. T. Tan, Polym. Chem., 2014, 5, 4054.
26 T. E. Kang, K. H. Kim and B. J. Kim, J. Mater. Chem. A, 2014, 2, 15252.

27 P. Shen, H. J. Bin, L. Xiao and Y. F. Li, Macromolecules, 2013, 46, 9575.

28 Y. S. Huang, M. Zhang, H. J. Chen, F. Wu, Z. C. Cao, L. J. Zhang and S. T. Tan, J. Mater. Chem. A, 2014, 2, 5218.

29 K. H. Hendriks, G. H. L. Heintges, V. S. Gevaerts, M. M. Wienk and R. A. J. Janssen, Angew. Chem., Int. Ed., 2013, 52, 8341.

30 Y. Zhang, J. Y. Zou, H. L. Yip, K. S. Chen, D. F. Zeigler, Y. Sun and A. K. Y. Jen, Chem. Mater., 2011, 23, 2289.

31 Q. P. Fan, Y. Liu, M. J. Xiao, H. Tan, Y. F. Wang, W. Y. Su, D. L. Yu, R. Q. Yang and W. G. Zhu, Org. Electron., 2014, 15, 3375 .

32 S. Li, Z. C. He, J. Yu, S. A. Chen, A. S. Zhong, R. L. Tang, H. B. Wu, J. G. Qin and Z. Li, J. Mater. Chem., 2012, 22, 12523.

33 J. Zhang, W. Z. Cai, F. Huang, E. G. Wang, C. M. Zhong, S. J. Liu, M. Wang, C. H. Duan, T. B. Yang and Y. Cao, Macromolecules, 2011, 44, 894.

34 L. Ye, S. Q. Zhang, W. C. Zhao, H. F. Yao and J. H. Hou, Chem. Mater., 2014, 26, 3603.

35 L. Ye, S. Q. Zhang, L. J. Huo, M. J. Zhang and J. H. Hou, Acc. Chem. Res., 2014, 47, 1595.

36 M. J. Zhang, Y. Gu, X. Guo, F. Liu, S. Q. Zhang, L. J. Huo, T. P. Russell and J. H. Hou, Adv. Mater., 2013, 25, 4944.

37 B. Liu, X. W. Chen, Y. H. He, Y. F. Li, X. J. Xu, L. Xiao, L. D. Li and Y. P. Zou, J. Mater. Chem. A, 2013, 1, 570.

38 K. Mahmood, H. Lu, Z. P. Liu, C. B. Li, Z. Lu, X. Liu, T. Fang, Q. H. Peng, G. W. Li, L. Li and Z. S. Bo, Polym. Chem., 2014, 5, 5037.

39 W. Lee, G. H. Kim, S. J. Ko, S. Yum, S. H Wang, S. Cho, Y. H. Shin, J. Y. Kim and H. Y. Woo, Macromolecules, 2014, 47, 1604.

40 G. W. Li, C. Kang, X. Gong, J. C. Zhang, C. H. Li, Y. C. Chen, H. L. Dong, W. P. Hu, F. H. Li and Z. S. Bo, Macromolecules, 2014, 47, 4645.

41 T. S. Qin, W. Zajaczkowski, W. Pisula, M. Baumgarten, M. Chen, M. Gao, G. Wilson, C. D. Easton, K. Müllen and S. E. Watkins, J. Am. Chem. Soc., 2014, 136, 6049.

42 P. C. Zhou, Z. G. Zhang, Y. F. Li, X. G. Chen and J. G. Qin, Chem. Mater., 2014, 26, 3495.

43 Y. Wang, F. Yang, Y. Liu, R. X. Peng, S. J. Chen and Z. Y. Ge, Macromolecules, 2013, 46, 1368.

44 D. F. Dang, W. C. Chen, S. Himmelberger, Q. Tao, A. Lundin, R. Q. Yang, W. G. Zhu, A. Salleo, C. Müller and E. G. Wang, Adv. Energy Mater., 2014, 4, 1400680.

45 Q. P. Fan, Y. Liu, P. G. Yang, W. Y. Su, M. J. Xiao, J. H. Chen, M. Li, X. D. Wang, Y. F. Wang, H. Tan, R. Q. Yang and W. G. Zhu, Org. Electron., 2015, 23, 124.

46 M. Wang, X. W. Hu, P. Liu, W. Li, X. Gong, F. Huang and Y. Cao, J. Am. Chem. Soc., 2011, 133, 9638. 